\title{
STRUCTURAL PROJECTIONS ON JBW*-TRIPLES
}

\author{
C. MARTIN EDWARDS AND GOTTFRIED T. RÜTTIMANN
}

\begin{abstract}
A linear projection $R$ on a Jordan*-triple $A$ is said to be structural provided that, for all elements $a, b$ and $c$ in $A$, the equality $\{R a b R c\}=R\{a R b c\}$ holds. A subtriple $B$ of $A$ is said to be complemented if $A=B+\operatorname{Ker}(B)$, where $\operatorname{Ker}(B)=\{a \in A:\{B a B\}=0\}$. It is shown that a subtriple of a $\mathrm{JBW}^{*}$-triple is complemented if and only if it is the range of a structural projection.

A weak* closed subspace $B$ of the dual $E^{*}$ of a Banach space $E$ is said to be an $N^{*}$-ideal if every weak* continuous linear functional on $B$ has a norm preserving extension to a weak* continuous linear functional on $E^{*}$ and the set of elements in $E$ which attain their norm on the unit ball in $B$ is a subspace of $E$. It is shown that a subtriple of a $\mathrm{JBW}^{*}$-triple $A$ is complemented if and only if it is an $\mathrm{N}^{*}$-ideal, from which it follows that complemented subtriples of $A$ are weak* closed, and structural projections on $A$ are weak* continuous and norm non-increasing. It is also shown that every $\mathrm{N}^{*}$-ideal in $A$ possesses a triple product with respect to which it is a $\mathrm{JBW}^{*}$-triple which is isomorphic to a complemented subtriple of $A$.
\end{abstract}

\section{Introduction}

In a recent paper Loos and Neher [18] introduced the notion of complementation in Jordan pairs and Jordan*-triples. For each element $a$ in a Jordan*-triple $A$ the quadratic mapping $Q(a)$ is defined, for all $b$ in $A$, by

$$
Q(a) b=\{a b a\} \text {. }
$$

The kernel $\operatorname{Ker}(B)$ of a subset $B$ of a Jordan*-triple $A$ is the subspace of $A$ consisting of elements which are annihilated by the quadratic mappings $Q(b)$ as $b$ runs through $B$. A subtriple $B$ of $A$ is said to be complemented if $A$ is the sum of $B$ and its kernel. Such a subtriple is an inner ideal. Provided that $A$ is anisotropic, that is, for an element $a$ in $A$, the vanishing of $\{a a a\}$ implies that of $a$, the sum is necessarily direct. Moreover, it can be shown that the linear projection $R$ on $A$ having $B$ as its range subtriple has the property that, for all elements $a$ in $A$, we have

$$
Q(R a)=R Q(a) R
$$

That is to say that the mapping $R$ is structural in the sense of Loos [17]. Conversely, the image of a structural projection on an anisotropic Jordan*-triple is a complemented subtriple.

Recently the authors [9] studied the normed vector space properties of subtriples of $\mathrm{JB}^{*}$-triples which are, of course, anisotropic Jordan*-triples. They showed that a norm closed subtriple of a $\mathrm{JB}^{*}$-triple is an inner ideal if and only if it has the unique Hahn-Banach extension property. In this paper the techniques used there are extended to the study of complementation of subtriples of $\mathrm{JBW}^{*}$-triples. For a subset $B$ of a Banach space $A$, Taylor [21] introduced the homogeneous subset $B^{\sharp}$ of the dual

Received 3 November 1993.

1991 Mathematics Subject Classification 46L10.

Research supported by the United Kingdom Science and Engineering Research Council and the Schweizerische Nationalfonds/Fonds national suisse. 
space $A^{*}$ of $A$ which is the set of elements of $A^{*}$ the restrictions of which to $B$ suffer no reduction in norm. A weak* closed subspace $B$ of a dual Banach space $E^{*}$ is said to be an $\mathrm{N}^{*}$-ideal if it has the properties that every weak* continuous linear functional on $B$ has a norm-preserving weak* continuous linear extension to $E^{*}$ and the intersection of $B^{\sharp}$ with the canonical image of $E$ in $E^{* *}$ is a subspace. The first main result of the paper is that a subtriple of a $\mathrm{JBW}^{*}$-triple is complemented if and only if it is an $\mathrm{N}^{*}$-ideal. This result can be considered as a generalization of results of Horn [14], Barton and Timoney [2], which imply that the weak* closed M-ideals in a JBW*triple are precisely its weak* closed ideals. In fact these authors showed that the Mideals in a $\mathrm{JB}^{*}$-triple coincide with its norm closed ideals. This result is also open to generalization. A closed subspace $B$ in a $\mathrm{JB}^{*}$-triple $A$ is said to be an $\mathrm{N}$-ideal provided that $B^{\sharp}$ is a subspace of the dual space $A^{*}$ of $A$. It is shown that a subtriple $B$ of a $\mathrm{JB}^{*}$-triple $A$ is an $\mathrm{N}$-ideal if and only if $B$ is an inner ideal in $A$, the second dual $B^{* *}$ of which, when identified with the second annihilator $B^{\circ \circ}$ in $A^{* *}$, is a complemented subtriple of the $\mathrm{JBW}^{*}$-triple $A^{* *}$.

It follows from the pathfinding work of $\mathrm{W}$. Kaup [16] that an $\mathrm{N}^{*}$-ideal $B$ in a $\mathrm{JBW}^{*}$-triple $A$ is always a $\mathrm{JBW}^{*}$-triple with respect to the triple product $\{\ldots\}_{B}$ defined, for elements $a, b$ and $c$ in $B$ by

$$
\{a b c\}_{B}=R\{a b c\}
$$

where $R$ is the structural linear projection from $A$ onto $B$. It is shown that this JBW*triple is isomorphic to a weak* closed subtriple of $A$ which, of course, coincides with $B$ when $B$ is itself a subtriple of $A$.

The paper is organized as follows. In $\S 2$ definitions are given, notation is established and certain preliminary results are described. In $\S 3$ the concepts of $\mathrm{N}$-ideals and $\mathrm{N}^{*}$-ideals are introduced and their properties, many of which are of independent interest, are investigated. In $\S 4$ the main result is stated and proved and $\S 5$ is devoted to a discussion of some further results on $\mathrm{N}^{*}$-ideals in $\mathrm{JBW}^{*}$-triples.

\section{Preliminaries}

A Jordan*-algebra $A$ which is also a complex Banach space such that, for all elements $a$ and $b$ in $A$, we have $\left\|a^{*}\right\|=\|a\|,\|a \circ b\| \leqslant\|a\|\|b\|$ and $\|\{a a a\}\|=\|a\|^{3}$, where

$$
\{a b c\}=a \circ\left(b^{*} \circ c\right)+\left(a \circ b^{*}\right) \circ c-b^{*} \circ(a \circ c)
$$

is the Jordan triple product on $A$, is said to be a Jordan $\mathrm{C}^{*}$-algebra [22] or JB*-algebra [23]. A Jordan $\mathrm{C}^{*}$-algebra which is the dual of a Banach space is said to be a Jordan $\mathrm{W}^{*}$-algebra [7] or a $\mathrm{JBW}^{*}$-algebra [23]. For the algebraic properties of Jordan algebras the reader is referred to $[15,19,20]$.

Recall that a complex vector space $A$ equipped with a triple product

$$
(a, b, c) \longmapsto\{a b c\}
$$

from $A \times A \times A$ to $A$ which is symmetric and linear in the first and third variables, conjugate linear in the second variable and satisfies the identity

$$
[D(a, b), D(c, d)]=D(\{a b c\}, d)-D\{c,\{d a b\})=D(a,\{b c d\})-D(\{c d a\}, b),
$$

where [ , ] denotes the commutator and $D$ is the mapping from $A \times A$ to $A$ defined by $D(a, b) c=\{a b c\}$, is said to be a Jordan*-triple. When $A$ is also a Banach space such 
that $D$ is continuous from $A \times A$ to the Banach space $B(A)$ of bounded linear operators on $A$ and, for each element $a$ in $A$, we have that $D(a, a)$ is hermitian with non-negative spectrum and satisfies $\|D(a, a)\|=\|a\|^{2}$, then $A$ is said to be a JB*triple. It can be shown that, if $a, b$ and $c$ are elements in a JB*-triple $A$, then $\|\{a b c\}\| \leqslant$ $\|a\|\|b\|\|c\|[11]$ and $\|\{a a a\}\|=\|a\|^{3}$ [16]. A JB*-triple which is the dual of a Banach space is said to be a $\mathrm{JBW}^{*}$-triple. Examples of $\mathrm{JB}^{*}$-triples are $\mathrm{JB}^{*}$-algebras and examples of $\mathrm{JBW}^{*}$-triples are $\mathrm{JBW}^{*}$-algebras. Isomorphisms of $\mathrm{JB}^{*}$-triples are automatically isometric and isomorphisms of $\mathrm{JBW}^{*}$-triples are automatically weak* continuous. For details see $[6,2]$. The second dual $A^{* *}$ of a $\mathrm{JB}^{*}$-triple $A$ possesses a triple product with respect to which it is a $\mathrm{JBW}^{*}$-triple, the canonical mapping from $A$ into $A^{* *}$ being an isomorphism. For details the reader is referred to $[5,6]$.

A subspace $J$ of a Jordan*-triple $A$ is said to be an inner ideal if $\{J A J\}$ is contained in $J$ and is said to be an ideal if $\{A A J\}+\{A J A\}$ is contained in $J$.

An element $u$ in a $\mathrm{JBW}^{*}$-triple $A$ is said to be a tripotent if $\{u u u\}$ is equal to $u$. The set of tripotents in $A$ is denoted by $\mathscr{U}(A)$. For each tripotent $u$ in the $\mathrm{JBW}^{*}$-triple $A$ the weak* continuous conjugate linear operator $Q(u)$ and the weak* continuous linear operators $P_{j}(u)$, for $j=0,1,2$, are defined by

$$
\begin{gathered}
Q(u) a=\{u a u\}, \quad P_{2}(u)=Q(u)^{2}, \\
P_{1}(u)=2\left(D(u, u)-Q(u)^{2}\right), \quad P_{0}(u)=I-2 D(u, u)+Q(u)^{2} .
\end{gathered}
$$

The linear operators $P_{j}(u)$ for $j=0,1,2$, are projections onto the eigenspaces $A_{j}(u)$ of $D(u, u)$ corresponding to eigenvalues $\frac{1}{2} j$ and

$$
A=A_{0}(u) \oplus A_{1}(u) \oplus A_{2}(u)
$$

is the Peirce decomposition of $A$ relative to $u$. For $i, j, k=0,1,2$ we have that $A_{i}(u)$ is a sub-JBW*-triple such that $\left\{A_{i}(u) A_{j}(u) A_{k}(u)\right\} \subseteq A_{i-j+k}(u)$ when $i-j+k=0,1$, or 2 , and $\{0\}$ otherwise, and

$$
\left\{A_{2}(u) A_{0}(u) A\right\}=\left\{A_{0}(u) A_{2}(u) A\right\}=\{0\} .
$$

Moreover, $A_{0}(u)$ and $A_{2}(u)$ are inner ideals in $A$ and $A_{2}(u)$ is a JBW*-algebra with respect to the product $(a, b) \mapsto\{a u b\}$, unit $u$ and involution $a \mapsto\{u a u\}$. A pair $u, v$ of elements of $\mathscr{U}(A)$ is said to be orthogonal if $v$ is contained in $A_{0}(u)$. For two elements $u$ and $v$ of $\mathscr{U}(A)$, write $u \leqslant v$ if $\{u v u\}=u$ or, equivalently, if $v-u$ is a tripotent orthogonal to $u$. This defines a partial ordering on $\mathscr{U}(A)$ with respect to which $\mathscr{U}(A)$ with a greatest element adjoined forms a complete lattice. For each element $a$ in the $\mathrm{JBW}^{*}$-triple $A$ there exists a unique tripotent $r(a)$ in $A$ called the support of $a$, being the smallest element of $\mathscr{U}(A)$ such that $a$ is a positive element in the $\mathrm{JBW}^{*}$-algebra $A_{2}(r(a))$.

Let $E$ be a complex Banach space. Recall that a linear projection $P$ on $E$ is said to be an L-projection if

$$
\|x\|=\|P x\|+\|x-P x\|
$$

for each element $x$ in $E$. A closed subspace which is the range of an L-projection is said to be an L-summand of $E$. Let $A$ be a complex Banach space. Recall that a linear projection $R$ on $A$ is said to be an M-projection if

$$
\|a\|=\sup \{\|R a\|,\|a-R a\|\}
$$

for each element $a$ in $A$. A closed subspace which is the range of an M-projection is said to be an M-summand of $A$. A closed subspace $B$ of $A$ is said to be an M-ideal if its annihilator $B^{\circ}$ in the dual space $A^{*}$ of $A$ is an L-summand in $A^{*}$. Clearly, every $\mathrm{M}$-summand is an $\mathrm{M}$-ideal. For details the reader is referred to $[\mathbf{1}, \mathbf{4}]$. 
Let $B$ be a weak* closed subtriple of the JBW*-triple $A$ and let

$$
B^{\perp}=\bigcap\left\{A_{0}(u): u \in \mathscr{U}(B)\right\} .
$$

Then $B^{\perp}$ is a weak* closed inner ideal in $A$, the sum $B+B^{\perp}$ is a weak* closed subtriple of $A$ and is an M-sum of $B$ and $B^{\perp}$ which are weak* closed ideals in $B \oplus_{M} B^{\perp}$. If $I$ is a weak* closed ideal in $A$ then so also is $I^{\perp}$ and

$$
A=I \oplus_{M} I^{\perp} \text {. }
$$

In fact the weak* closed ideals in $A$ coincide with its M-summands. Moreover the set of M-ideals in a $\mathrm{JB}^{*}$-triple $A$ coincides with the set of norm closed ideals in $A$ $[2,3,14,20]$.

\section{Structure in complex Banach spaces}

Throughout this section the convention of identifying a Banach space with its canonical image in its second dual space will be adopted. In particular, the second dual $B^{* *}$ of a subspace $B$ of a Banach space $E$ will be identified with the second annihilator $B^{\circ \circ}$ of $B$ in the second dual $E^{* *}$ of $E$.

Let $E$ be a complex Banach space. A linear projection $P$ on $E$ is said to be neutral [13] if $\|P x\| \leqslant\|x\|$ for all elements $x$ in $E$, and if $x$ is an element of $E$ for which $\|P x\|=\|x\|$ then $P x=x$. Notice that L-projections are neutral.

Let $E$ be a complex Banach space and let $B$ be a subspace of $E$. Define the subset $B^{\sharp}$ of the dual space $E^{*}$ of $E$ by

$$
B^{\sharp}=\left\{x \in E^{*}:\|x\|=\sup _{a \in B_{1}}|x(a)|\right\},
$$

where $B_{1}$ denotes the unit ball in $B$ [21]. Notice that $B^{\sharp}$ is a homogeneous though, in general, non-linear subset of $E^{*}$. Moreover, if $\bar{B}^{n}$ denotes the norm closure of $B$ then $\left(\bar{B}^{n}\right)^{\sharp}$ coincides with $B^{\sharp}$. Suppose that $A$ is the dual space of the complex Banach space $E$, let $B$ be a subspace of $A$, and let $\vec{B}^{\omega^{*}}$ denote the weak* closure of $B$. Observe that the subsets $\left(\vec{B}^{w *}\right)^{\sharp} \cap E$ and $B^{\sharp} \cap E$ of $E$ coincide.

\section{Lemma 3.1. Let $P$ be a neutral projection on a Banach space E. Then}

$$
\operatorname{im} P=\left(\operatorname{im} P^{*}\right)^{\sharp} \cap E .
$$

Proof. First notice that since $P$ is norm non-increasing it follows that the sets (im $\left.P^{*}\right)_{1}$ and $P^{*}\left(E_{1}^{*}\right)$ coincide. Since im $P$ coincides with the annihilator $\left(\operatorname{ker} P^{*}\right)_{\circ}$ of the kernel $\operatorname{ker} P^{*}$ of $P^{*}$, for each element $x$ in $\operatorname{im} P$ we have

$$
\|x\|=\sup _{a \in E_{1}^{*}}|x(a)|=\sup _{a \in E_{1}^{*}}\left|x\left(P^{*} a\right)+x\left(a-P^{*} a\right)\right|=\sup _{a \in E_{1}^{*}}\left|x\left(P^{*} a\right)\right|=\sup _{a \in\left(\operatorname{Im} P^{*}\right)_{1}}|x(a)| .
$$

Consequently im $P^{*}$ is contained in $\left(\mathrm{im} P^{*}\right)^{\sharp} \cap E$. Conversely, if $x$ is an element in $\left(\mathrm{im} P^{*}\right)^{\sharp} \cap E$, then

$$
\|x\|=\sup _{a \in\left(\operatorname{Im} P P^{*}\right)}|x(a)|=\sup _{a \in E_{1}^{*}}\left|x\left(P^{*} a\right)\right|=\|P x\| .
$$

Since $P$ is neutral it follows that $P x$ and $x$ are equal and this implies that $x$ is contained in $\operatorname{im} P$. 
Lemma 3.2. Let $P$ be a neutral projection on a Banach space E. Then

$$
E^{*}=\operatorname{im} P^{*} \oplus\left(\left(\operatorname{im} P^{*}\right)^{\sharp} \cap E\right)^{\circ}, \quad E=\left(\left(\operatorname{im} P^{*}\right)^{\sharp} \cap E\right) \oplus\left(\operatorname{im} P^{*}\right)_{\circ} .
$$

Proof. Since ker $P^{*}$ coincides with $(\mathrm{im} P)^{\circ}$ and since $\operatorname{ker} P$ coincides with (im $\left.P^{*}\right)_{\circ}$ the result follows from Lemma 3.1.

LEMma 3.3. Let $P$ and $Q$ be neutral projections on a Banach space $E$. Then $P=Q$ if and only if $\operatorname{im} P^{*}=\operatorname{im} Q^{*}$.

Proof. Let $\operatorname{im} P^{*}=\operatorname{im} Q^{*}$. Since $\operatorname{ker} P=\left(\operatorname{im} P^{*}\right)_{\circ}=\left(\operatorname{im} Q^{*}\right)_{\circ}=\operatorname{ker} Q$ and, by Lemma $3.1, \operatorname{im} P=\left(\operatorname{im} P^{*}\right)^{\sharp} \cap E=\left(\operatorname{im} Q^{*}\right)^{\#} \cap E=\operatorname{im} Q$ it follows that $P$ and $Q$ coincide (cf. [13, Lemma 2.2]).

LEMMA 3.4. Let $E$ be a Banach space and let $B$ be a subspace of the dual space $E^{*}$ of $E$ having the property that every weak* continuous linear functional on $B$ has a norm preserving extension to a weak* continuous linear functional on $E^{*}$. Then

$$
E=\left(B^{\sharp} \cap E\right)+B_{\circ}, \quad\left(B^{\sharp} \cap E\right) \cap B_{\circ}=\{0\} .
$$

Proof. Let $x$ be an element in $E$. By hypothesis there exists an element $y$ in $E$ such that the restriction $\left.y\right|_{B}$ of $y$ to $B$ and the restriction $\left.x\right|_{B}$ of $x$ to $B$ coincide and $\|y\|=\left\|\left.x\right|_{B}\right\|$. Then $\|y\|=\sup _{a \in B_{1}}|x|_{B}(a)\left|=\sup _{a \in B_{1}}\right| y(a) \mid$ and $y$ is contained in $B^{\sharp} \cap E$. Clearly the element $x-y$ lies in $B_{\circ}$ and the first part of the lemma follows. Finally, if $x$ is an element in $\left(B^{\sharp} \cap E\right) \cap B_{\circ}$ then $\|x\|=\sup _{a \in B_{1}}|x(a)|=0$ as required.

A weak* closed subspace $B$ of the dual space $E^{*}$ of the Banach space $E$ is said to be an $\mathrm{N}^{*}$-ideal if every weak* continuous linear functional on $B$ has a norm preserving extension to a weak* continuous linear functional on $E^{*}$ and the subset $B^{\sharp} \cap E$ of $E$ is a subspace. The next results show that there is an intimate connection between neutral projections on $E$ and $\mathrm{N}^{*}$-ideals in $E^{*}$.

Lemma 3.5. Let $P$ be a neutral projection on a Banach space $E$. Then the range im $P^{*}$ of the adjoint $P^{*}$ of $P$ is an $\mathrm{N}^{*}$-ideal.

Proof. By Lemma 3.1, the subset (im $\left.P^{*}\right)^{\sharp} \cap E$ is a subspace of $E$. Suppose that $y$ is a weak* continuous linear functional on im $P^{*}$. Define the weak* continuous linear functional $x$ on $E^{*}$, for each element $a$ in $E^{*}$, by $x(a)=y\left(P^{*} a\right)$. Then $x$ is an extension of $y$ to $E^{*}$ and, since $\left(\operatorname{im} P^{*}\right)_{1}$ and $P^{*}\left(E_{1}^{*}\right)$ coincide,

$$
\|x\|=\sup _{a \in E_{1}^{*}}|x(a)|=\sup _{a \in E_{1}^{*}}\left|y\left(P^{*} a\right)\right|=\sup _{a \in\left(\operatorname{im} P^{*}\right)_{1}}|y(a)|=\|y\| .
$$

Lemma 3.6. Let $B$ be an $\mathrm{N}^{*}$-ideal in the dual space $E^{*}$ of a Banach space $E$.

(i) Every weak* continuous linear functional on $B$ has a unique norm preserving extension to a weak* continuous linear functional on $E^{*}$.

(ii) There exists a unique neutral projection $P$ on $E$ such that the kernel of $P$ is equal to the annihilator $B_{0}$ of $B$ in $E$. In this case im $P^{*}$ coincides with $B$ and $\operatorname{im} P$ coincides with $B^{\sharp} \cap E$. 
Proof. By hypothesis, every weak* continuous linear functional on $B$ possesses a norm preserving weak* continuous linear extension to $E^{*}$. Suppose that $x$ and $y$ are elements of $E$ the restrictions of which to $B$ are equal and such that

$$
\left\|\left.x\right|_{B}\right\|=\|x\|, \quad\left\|\left.y\right|_{B}\right\|=\|y\| .
$$

Then $x$ and $y$ lie in the set $B^{\sharp} \cap E$ and therefore, by hypothesis, so does $x-y$. But $x-y$ lies in $B_{\circ}$ and it follows from Lemma 3.4 that $x$ and $y$ are equal, thereby completing the proof of (i).

For an element $x$ in $E$, let $P x$ denote the unique norm preserving weak* continuous linear extension to $E^{*}$ of the restriction $\left.x\right|_{B}$ of $x$ to $B$. Then, clearly $P(P x)$ and $P x$ are equal, $P x$ lies in $B^{\sharp} \cap E$, and $\|P x\|=\left\|\left.x\right|_{B}\right\| \leqslant\|x\|$. For elements $x$ and $y$ in $E$, since $P x+P y$ lies in $B^{\sharp} \cap E$, we have

$$
\|P x+P y\|=\sup _{a \in B_{1}}|(P x+P y)(a)|=\sup _{a \in B_{1}}|(P(x+y))(a)|=\|P(x+y)\| .
$$

Therefore, the elements $P x+P y$ and $P(x+y)$ of $E$ have the same norm. But they also have the same restrictions to $B$ and it follows from (i) that they coincide. Similarly, for each complex number $t$, the elements $t(P x)$ and $P(t x)$ are equal. Therefore $P$ is a norm non-increasing linear projection on $E$.

Let $x$ be an element of $E$ such that $\|P x\|$ is equal to $\|x\|$. Since $P x$ and $x$ have the same restrictions to $B$ it follows from (i) that $P x$ and $x$ are equal and hence that $P$ is neutral.

Suppose now that $x$ lies in $B_{\circ}$. Then, since $\|P x\|=\left\|\left.x\right|_{B}\right\|=0$, it follows that $x$ is contained in the kernel ker $P$ of $P$. On the other hand if $x$ is contained in $\operatorname{ker} P$, then $\left.x\right|_{B}=\left.(P x)\right|_{B}=0$ and $x$ lies in $B_{0}$. Therefore the kernel of $P$ and $B_{0}$ coincide. Uniqueness of the neutral projection follows from Lemma 3.3.

Finally, observe that, since $B$ is weak* closed, im $P^{*}$ coincides with $B$ and therefore, by Lemma 3.1, im $P$ coincides with $B^{\sharp} \cap E$.

It is now possible to make precise the connection between neutral projections and $\mathrm{N}^{*}$-ideals.

THEOREM 3.7. Let $E$ be a complex Banach space. Then the mapping $P \mapsto \operatorname{im} P^{*}$ is a bijection from the set of neutral projections on $E$ onto the set of $\mathrm{N}^{*}$-ideals in the dual space $E^{*}$ of $E$.

Proof. This follows from Lemma 3.5, Lemma 3.6(ii) and Lemma 3.3.

This result reveals a certain duality between neutral projections on a Banach space and $\mathrm{N}^{*}$-ideals in its dual. Attention is now turned to a similar duality which exists between certain subspaces of a Banach space and neutral projections on its dual.

Lemma 3.8. Let $B$ be a subspace of a Banach space $E$. Then

$$
E^{*}=B^{\sharp}+B^{\circ}, \quad B^{\sharp} \cap B_{\circ}=\{0\} .
$$

Proof. By the Hahn-Banach theorem, for each element $x$ in $E^{*}$, there exists an element $y$ in $E^{*}$ such that both $x$ and $y$ have the same restrictions to $B$ and $\|y\|=\left\|\left.x\right|_{B}\right\|$. Clearly $y$ is contained in $B^{\sharp}$ and $x-y$ is contained in $B^{\circ}$. Finally, if $x$ lies in $B^{\sharp} \cap B^{\circ}$, then, as in the proof of Lemma 3.4, necessarily $x$ is zero. 
A norm closed subspace $B$ of a Banach space $E$ is said to be an $\mathrm{N}$-ideal if the subset $B^{\sharp}$ of the dual space $E^{*}$ of $E$ is a subspace.

LEMMA 3.9. Let $B$ be an $\mathrm{N}$-ideal in the complex Banach space $E$.

(i) Every bounded linear functional on $B$ has a unique norm preserving extension to a bounded linear functional on $E$.

(ii) There exists a unique neutral projection $P$ on the dual space $E^{*}$ of $E$ such that the kernel ker $P$ of $P$ coincides with the annihilator $B^{\circ}$ of $B$ in $E^{*}$. In this case im $P^{*} \cap E$ coincides with $B$ and im $P$ coincides with $B^{\sharp}$.

Proof. By the Hahn-Banach theorem a bounded linear functional on $B$ possesses a norm preserving extension to a bounded linear functional on $E$. As in the proof of Lemma 3.6(i), using Lemma 3.8 in place of Lemma 3.4, it follows that the extension is unique.

For each element $x$ in $E^{*}$ define $P x$ to be the unique norm preserving linear extension to $E$ of the restriction of $x$ to $B$. Then, as in the proof of Lemma 3.6(ii), it can be seen that $P$ is a neutral projection on $E^{*}$, that $\operatorname{ker} P$ coincides with $B^{\circ}$ and that im $P$ is contained in $B^{\sharp}$. Uniqueness of the neutral projection follows, by Lemma 3.3.

Suppose that $x$ is an element in $B^{\sharp}$. Then, since $P x$ and $x$ have the same restriction to $B$, it follows that $\|P x\|=\left\|\left.x\right|_{B}\right\|=\|x\|$ which implies that $P x$ equals $x$. Therefore, the range im $P$ of $P$ is $B^{\sharp}$. By Theorem 3.7 it can be seen that the range im $P^{*}$ of $P^{*}$ is an $\mathrm{N}^{*}$-ideal in $\mathrm{E}^{* *}$. Moreover, im $P^{*} \cap E=(\operatorname{ker} P)^{\circ} \cap E=B^{\circ \circ} \cap E=B$ and the uniqueness of $P$ follows from Theorem 3.7.

The proof above immediately verifies the following result.

COROllary 3.10. Let $B$ be an $\mathrm{N}$-ideal in the complex Banach space E. Then $B^{\circ o}$ is an $\mathrm{N}^{*}$-ideal in $E^{* *}$.

\section{Structure in $\mathrm{JB}^{*}$-triples and $\mathrm{JBW}^{*}$-triples}

Let $A$ be a Jordan*-triple. Let $B$ be a linear subspace of $A$ and define the kernel of $B[\mathbf{1 8}]$ to be the linear subspace

$$
\operatorname{Ker}(B)=\{a \in A:\{B a B\}=0\} .
$$

A subtriple $B$ of the Jordan*-triple $A$ is said to be complemented if

$$
A=B+\operatorname{Ker}(B) \text {. }
$$

Let $u$ be a tripotent in $A$. Then $\operatorname{Ker}\left(A_{2}(u)\right)=\operatorname{ker} Q(u)=A_{1}(u)+A_{0}(u)$ and therefore $A_{2}(u)$ is a complemented subtriple of $A$.

Let $A$ be a Jordan* triple. A linear projection $R: A \rightarrow A$ is said to be a structural projection if $Q(R a)=R Q(a) R$ for all elements $a$ in $A$, or, equivalently, $\{R a b R c\}=$ $R\{a R b c\}$ for all elements $a, b$ and $c$ in $A$. It is easily verified that, for every tripotent $u$ in $A$, the Peirce projections $P_{2}(u)$ and $P_{0}(u)$ are a structural projections.

Lemma 4.1. Let $B$ be a complemented subtriple of a Jordan*-triple $A$. Then $B$ is an inner ideal in $A$ and the subset $\{A B \operatorname{Ker}(B)\}$ of $A$ is contained in $\operatorname{Ker}(B)$. 
Proof. Notice that

$$
\{B A B\}=\{B B+\operatorname{Ker}(B) B\} \subseteq B+\{B \operatorname{Ker}(B) B\}=B
$$

from which it follows that $B$ is an inner ideal in $A$. Now let $a, b$ and $c$ be elements of $B$, let $d$ be an element in $A$ and let $e$ be an element of $\operatorname{Ker}(B)$. Then, by the Jordan triple identity,

$$
\begin{aligned}
\{a\{d b e\} c\} & =\{\{b e a\} d c\}+\{\text { ad }\{b e c\}\}-\{b e\{a d c\}\} \\
& \subseteq\{\{0\} A B\}+\{B A\{0\}\}+\{B \operatorname{Ker}(B) B\}=\{0\} .
\end{aligned}
$$

It follows that the element $\{d b e\}$ lies in $\operatorname{Ker}(B)$.

Attention is now turned to $\mathrm{JBW}^{*}$-triples. The first two results summarize straightforward properties of the formation of the kernel of a subset.

Lemma 4.2. Let $A$ be $a \mathrm{JBW}^{*}$-triple and let $B$ be a subspace of $A$. $\vec{B}^{w^{*}}$ of $B$.

(ii) The kernel $\operatorname{Ker}(B)$ of $B$ is a weak* closed subspace of $A$.

(iii) The intersection of $B$ and $\operatorname{Ker}(B)$ is $\{0\}$.

Proof. Statements (i) and (ii) follow from the separate weak* continuity of the triple product on $A$ and (iii) follows from the anisotropicity of JB*-triples.

LEMMA 4.3. Let $B$ be a complemented subtriple of $\mathrm{JBW}^{*}$-triple $A$. Then $B$ is $a$ weak* closed inner ideal in $A$.

Proof. That $B$ is an inner ideal follows from Lemma 4.1. Moreover, using Lemma 4.2,

$$
A=B \oplus \operatorname{Ker}(B) \subseteq \vec{B}^{w^{*}} \oplus \operatorname{Ker}(B) \subseteq A
$$

It follows that $B$ and $\vec{B}^{w^{*}}$ coincide.

LEMMA 4.4. Let $R$ be a structural projection on a JBW*-triple $A$. Then the range $\operatorname{im} R$ of $R$ is a complemented subtriple of $A$ and

$$
\operatorname{Ker}(\operatorname{im} P)=\operatorname{ker} P \text {. }
$$

Proof. Let $R$ be a structural projection on $A$. Then $\operatorname{im} R$ is clearly an inner ideal in $A$. Moreover, if $b$ lies in the kernel $\operatorname{ker} R$ of $R$ then, for all elements $a$ and $c$ in im $R$, we have

$$
\{a b c\}=\{R a b R c\}=R\{a R b c\}=0
$$

and it follows that $\operatorname{ker} R$ is contained in $\operatorname{Ker}(\operatorname{im} R)$. Conversely, if $a$ lies in $\operatorname{Ker}(\operatorname{im} R)$ then

$$
\{R a R a R a\}=R\{R a R a R a\}=\{R a a R a\}=0
$$

and it follows that $R a$ is zero. Hence $\operatorname{ker} R$ and $\operatorname{Ker}(\operatorname{im} R)$ coincide. Since

$$
A=\operatorname{im}(R)+\operatorname{ker} R=\operatorname{im} R+\operatorname{Ker}(\operatorname{im} R)
$$

it follows that $\operatorname{im} R$ is a complemented subtriple of $A$. 
THEOREM 4.5. Let $A$ be a JBW*-triple. The mapping $R \mapsto \operatorname{im} R$ is a bijection from the set of structural projections on $A$ onto the set of complemented subtriples of $A$.

Proof. Let $B$ be a complemented subtriple of $A$. Let $R$ be the linear projection on $A$ with range $B$ and kernel $\operatorname{Ker}(B)$. Using Lemma 4.1, observe that, for elements $a$ and $b$ in $A$, the elements $\{R a R b(a-R a)\}$ and $\{(a-R a) R b(a-R a)\}$ lie in $\operatorname{Ker}(B)$. Therefore, using this and the hypothesis that $B$ is a subtriple,

$$
\begin{aligned}
R\{a R b a\} & =R\{(R a+(a-R a)) R b(R a+(a-R a))\}=R\{R a R b R a\} \\
& =\{R a R b R a\}=\{R a(b-(b-R b)) R a\}=\{R a b R a\} .
\end{aligned}
$$

It follows that $R$ is a structural projection with range $B$. Suppose that $Q$ is a further structural projection with the same range. Then, by Lemma 4.4, $\operatorname{ker} R=\operatorname{Ker}(\operatorname{im} R)=\operatorname{Ker}(\operatorname{im} Q)=\operatorname{ker} Q$, and therefore $Q$ is equal to $R$.

Lemma 4.6. Let $A$ be a $\mathrm{JBW}^{*}$-triple, let $A_{*}$ be the predual of $A$ and let $B$ be $a$ subtriple of $A$. Then

$$
\operatorname{Ker}(B)=\bigcap_{u \in \mathscr{U}\left(B^{w^{*}}\right)} \operatorname{ker} P_{2}(u)=\bigcap_{b \in B} \operatorname{ker} P_{2}(r(b))
$$

where $r(b)$ denotes the support tripotent of an element $b$ in $A$.

Proof. By [8, Lemma 3.1], for each element $b$ in $B$, the support tripotent $r(b)$ is the weak* limit of a sequence of real odd polynomials in $b$. Therefore $r(b)$ is contained in the $\mathrm{JBW}^{*}$-triple $\vec{B}^{w^{*}}$. Let $a$ be an element of $A$ such that $P_{2}(r(b)) a$ is zero for all elements $b$ in $B$. It follows that $\{r(b) \operatorname{ar}(b)\}$ is zero for all $b$ in $B$. Therefore,

$$
\{b a b\}=\{\{r(b) b r(b)\} a\{r(b) b r(b)\}\}=\{r(b)\{b\{r(b) a r(b)\} b\} r(b)\}=0
$$

and $a$ is contained in $\operatorname{Ker}(B)$. It is clear that $P_{2}(u) a$ is zero for every tripotent $u$ in $\bar{B}^{\omega^{*}}$ and $a$ in $\operatorname{Ker}\left(\vec{B}^{w^{*}}\right)$, which coincides with $\operatorname{Ker}(B)$. This completes the proof.

The next main result describes the connection between complemented subtriples of a JBW*-triple and its $\mathrm{N}^{*}$-ideals.

THeORem 4.7. Let $A$ be a JBW*-triple with predual $A_{*}$.

(i) Let $B$ be a subtriple of $A$. Then $B$ is complemented if and only if $B$ is an $\mathrm{N}^{*}$-ideal in $A$.

(ii) Let $P$ be a neutral projection on $A_{*}$. If the range im $P^{*}$ of the adjoint $P^{*}$ of $P$ is a subtriple of $A$ then $P^{*}$ is a structural projection on $A$.

(iii) The mapping $P \mapsto P^{*}$ is a bijection from the set of neutral projections on $A_{*}$ for each of which im $P^{*}$ is a subtriple onto the collection of structural projections on $A$.

Proof. Suppose that $B$ is a complemented subtriple in $A$. Then, by Lemma 4.3, $B$ is a weak* closed inner ideal in $A$ and, by [9, Theorem 2.6], every weak* continuous 
linear functional on $B$ has a norm preserving extension to a weak* continuous linear functional on $A$. Now let $x$ be an element of $B^{\sharp} \cap A_{*}$. Since the restriction $\left.x\right|_{B}$ of $x$ to $B$ is a weak* continuous linear functional on the subtriple $B$ of $A$, by $[\mathbf{1 0}$, Proposition 2], there exists a tripotent $u$ in $B$ such that $\|x\|=\|x \mid B\|=u(x)$. Then

$$
\|x\|=\left(P_{2}(u) u\right)(x)=u\left(P_{2}(u)_{*} x\right) \leqslant\left\|P_{2}(u)_{*} x\right\| \leqslant\|x\|,
$$

where $P_{2}(u)_{*}$ denotes the norm non-increasing projection on $A_{*}$ the adjoint of which is $P_{2}(u)$. By [12, Proposition 1], the element $x$ is contained in the range im $P_{2}(u)_{*}$. Since this range coincides with the annihilator $\left(\operatorname{ker} P_{2}(u)\right)_{\circ}$ of the kernel $\operatorname{ker} P_{2}(u)$ of $P_{2}(u)$, by Lemma 4.6,

$$
B^{\sharp} \cap A_{*} \subseteq \bigcup_{u \in \mathscr{U}(B)}\left(\operatorname{ker} P_{2}(u)\right)_{\circ} \subseteq\left(\bigcap_{u \in \mathscr{U}(B)} \operatorname{ker} P_{2}(u)\right)_{\circ}=\operatorname{Ker}(B)_{\circ} .
$$

Since $A$ is the direct sum of $B$ and $\operatorname{Ker}(B)$ it is clear that $B_{\circ} \cap \operatorname{Ker}(B)_{\circ}$ is zero. By Lemma 3.4,

$$
A_{*}=B^{\sharp} \cap A_{*}+B_{\circ} \subseteq \operatorname{Ker}(B)_{\circ} \oplus B_{\circ} \subseteq A_{*} .
$$

Therefore the set $B^{\sharp} \cap A_{*}$ coincides with the subspace $\operatorname{Ker}(B)_{0}$. It follows that $B$ is an $\mathrm{N}^{*}$-ideal in $A$.

Conversely, suppose that $B$ is a subtriple of $A$ which is an $\mathrm{N}^{*}$-ideal. By Lemma 3.6 and [9, Theorem 2.6], $B$ is a weak*-closed inner ideal in $A$. By the argument used above it can be seen that $\operatorname{Ker}(B)$ is contained in the annihilator $\left(B^{\sharp} \cap A_{*}\right)^{\circ}$ of the subspace $B^{\sharp} \cap A_{*}$ of $A_{*}$. Let $u$ be a tripotent in $B$ and let $x$ be an element of im $P_{2}(u)_{*}$. Since the unit ball $A_{1}$ in $A$ is weak* compact there exists an element $a$ in $A_{1}$ at which $x$ attains its norm. Then

$$
\|x\|=a(x)=a\left(P_{2}(u)_{*} x\right)=\left(P_{2}(u) a\right)(x) .
$$

But since $B$ is an inner ideal, the element $P_{2}(u) a$ is contained in $B$ and it follows that $x$ lies in the subspace $B^{\sharp} \cap A_{*}$ of $A_{*}$. Since im $P_{2}(u)_{*}$ coincides with ker $P_{2}(u)_{*}$ it follows that $\operatorname{ker} P_{2}(u)_{\circ}$ is contained in $B^{\sharp} \cap A_{*}$. Therefore, since $\operatorname{ker} P_{2}(u)$ is weak* closed,

$$
\left(B^{\sharp} \cap A_{*}\right)^{\circ} \subseteq\left(\sum_{u \in \mathscr{U}(A)}\left(\operatorname{ker} P_{2}(u)\right)_{\circ}\right)^{\circ} \subseteq \operatorname{Ker}(B) .
$$

By Theorem 3.7, there exists a neutral projection $P$ on $A_{*}$ such that $B$ coincides with im $P^{*}$. By Lemma 3.2,

$$
A=\operatorname{im} P^{*} \oplus\left(\left(\operatorname{im} P^{*}\right)^{\sharp} \cap A_{*}\right)^{\circ}=B \oplus\left(B^{\sharp} \cap A_{*}\right)^{\circ} \subseteq B \oplus \operatorname{Ker}(B) \subseteq A .
$$

It follows that $A$ is the direct sum of $B$ and $\operatorname{Ker}(B)$ and hence that $B$ is complemented. By the same token, it follows that

$$
\operatorname{Ker}\left(\operatorname{im} P^{*}\right)=\left(\left(\operatorname{im} P^{*}\right)^{\sharp} \cap A_{*}\right)^{\circ}=(\operatorname{im} P)^{\circ}=\operatorname{ker} P^{*} .
$$

Therefore, by Lemma 4.4 and Theorem $4.5, P^{*}$ is a structural projection on $A$.This completes the proof of (i) and (ii).

Let $R$ be a structural projection on $A$. By Lemma 4.4 and (i), im $R$ is an $\mathrm{N}^{*}$-ideal in $A$. Then there exists, by Corollary 3.7, a neutral projection $P$ on $A_{*}$ such that im $P^{*}$ coincides with im $R$. By Lemma 4.4, im $P^{*}$ is a subtriple of $A$ and therefore, by (ii), $P^{*}$ is a structural projection. It follows, by Theorem 4.5 , that $R$ is equal to $P$. This proves (iii). 
This theorem has several important corollaries which stem from the properties of $\mathrm{N}^{*}$-ideals and the fact that every complemented subtriple is the range of a unique structural projection.

COROllary 4.8. Let $A$ be a $\mathrm{JBW}^{*}$-triple and let $R$ be a structural projection on $A$. Then $R$ is norm non-increasing and weak* continuous.

COROllary 4.9. Let $B$ be a complemented subtriple of the $\mathrm{JBW}^{*}$-triple $A$ and let $A_{*}$ be the predual of $A$. Then $B$ is weak* closed and has as its predual the space $B^{\sharp} \cap A_{*}$.

Next we turn our attention to the study of $\mathrm{N}$-ideals in $\mathrm{JB}^{*}$-triples.

THEOREM 4.10. Let $A$ be a $J B^{*}$-triple and let $B$ be a norm closed subtriple of $A$. Then $B$ is an $\mathrm{N}$-ideal in $A$ if and only if the bi-annihilator $B^{\circ \circ}$ of $B$ is a complemented subtriple of the $\mathrm{JBW}^{*}$-triple $A^{* *}$.

Proof. Let $B$ be a norm closed subtriple of $A$ such that $B^{\circ \circ}$ is a complemented subtriple of $A^{* *}$. Then, there exists a unique neutral projection $P$ on $A^{*}$ with range $\left(\mathrm{B}^{\circ \circ}\right)^{\sharp} \cap E$. Since the canonical image of $B$ in $A^{* *}$ is a weak ${ }^{*}$ dense subspace of $B^{\circ \circ}$ the remarks preceding Lemma 3.1 show that the range of $P$ is the set $B^{\sharp}$. Therefore $B$ is an $\mathrm{N}$-ideal in $A$.

Conversely, suppose that the subtriple $B$ is an $\mathrm{N}$-ideal in $A$. By Corollary 3.10 and Theorem 4.7(i), the weak* closed subtriple $B^{\circ \circ}$ of $A^{* *}$ is complemented.

Corollary 4.11. Let $A$ be a JB*-triple. An $\mathrm{N}$-ideal $B$ in $A$ which is also a subtriple of $A$ is an inner ideal in $A$.

Proof. By Lemma 3.9, every bounded linear functional on $B$ has a unique norm preserving extension to a linear functional on $A$. The result follows from [9, Theorem 2.6].

Corollary 4.12. Let $A$ be $a \mathrm{JBW}^{*}$-triple and let $B$ be a weak* closed subtriple of $A$ which is an N-ideal in $A$. Then $B$ is a complemented subtriple of $A$.

Proof. By Corollary 4.11 it follows that $B$ is a weak* closed inner ideal in $A$. By [9, Theorem 2.5], every weak* continuous linear functional on $B$ has a norm preserving extension to a weak* continuous linear functional on $A$. Moreover, since $B^{\sharp}$ is a subspace of $A^{*}$ it follows that $B^{\sharp} \cap A_{*}$ is a subspace of $A_{*}$. Therefore, $B$ is an $\mathrm{N}^{*}$-ideal in $A$ as required.

\section{5. $\mathrm{N}^{*}$-ideals in $\mathrm{JBW}^{*}$-triples}

Recall that, for a $\mathrm{JBW}^{*}$-triple $A$ and an element $x$ in the predual $A_{*}$ of $A$ there exists a unique tripotent $e^{A}(x)$ in $A$ such that

$$
x\left(e^{A}(x)\right)=\|x\|
$$


and $x$ is a faithful normal positive linear functional on the $\mathrm{JBW}^{*}$-algebra $A_{2}\left(e^{A}(x)\right)$ [10]. The tripotent $e^{A}(x)$ is said to be the support of $x$ in $A$. Now let $P$ be a norm nonincreasing projection on the predual $A_{*}$ of $A$ and let $P^{*}$ be its adjoint. Then the work of W. Kaup [16] shows that the range $B$ of $P^{*}$ is a JBW*-triple with respect to the triple product $\{\ldots\}_{B}$ defined for elements $a, b$ and $c$ in $B$ by $\{a b c\}_{B}=P^{*}\{a b c\}$. Let $C$ be the smallest weak* closed subspace of $A$ containing the set $\left\{e^{A}(x): x \in P A_{*}\right\}$ and let $D$ be the weak* closed subtriple of $A$ which is the intersection of the family $\left(A_{0}\left(e^{A}(x)\right)\right)_{x \in P A}$ of weak* closed subtriples of $A$.

The following lemma represents a $\mathrm{JBW}^{*}$-triple version of results due to Friedman and Russo [12].

LEMMA 5.1. Let $A$ be a $\mathrm{JBW}^{*}$-triple, let $P$ be a norm non-increasing projection on the predual $A_{*}$ of $A$, let $B$ be the range of the weak ${ }^{*}$ continuous projection $P^{*}$ on $A$ and let the weak* closed subspace $C$ and the weak* closed subtriple $D$ of $A$ be defined as above.

(i) The spaces $C$ and $C+D$ are weak* closed subtriples of $A$ and the JBW *-triple $C+D$ is the M-sum of $C$ and $D$ in which $C$ and $D$ are weak ${ }^{*}$ closed ideals.

(ii) The set $B$ is contained in $C \oplus_{M} D$ and the restriction to $B$ of the M-projection $Q$ from $C \oplus_{M} D$ onto $C$ is a weak ${ }^{*}$ continuous isometric isomorphism from the JBW*triple $B$ endowed with the triple product $\{\ldots\}_{B}$ onto the $\mathrm{JBW}^{*}$-triple $C$.

(iii) The weak* closed subtriple $D$ coincides with the intersection of the family $\left(A_{0}(u)\right)_{u \in \mathscr{\Psi}(C)}$ of subtriples of $A$.

Proof. Notice that each tripotent $u$ in $D$ is contained in $A_{0}\left(e^{A}(x)\right)$, for all $x$ in $P A_{*}$. Then, for each element $x$ in $P A_{*}$, the tripotent $e^{A}(x)$ is contained in $A_{0}(u)$. Hence, for each tripotent $u$ in $D$, the weak* closed subspace $C$ is contained in $A_{0}(u)$. Consequently, $C$ is contained in $D^{\perp}$. However, since the $\mathrm{JBW}^{*}$-triple $D+D^{\perp}$ is an M-sum of $D$ and $D^{\perp}$ it follows that $C+D$ is an M-sum of weak* closed subspaces of $A$. It follows, by the Theorem of Krein-Šmulian, that $C \oplus_{M} D$ is weak* closed. Moreover, the M-projection $Q$ of $C \oplus_{M} D$ onto $C$ is weak* continuous. It follows from [12, Lemma 2.6] and its proof, that $B$ is contained in $C \oplus_{M} D$ and that $Q$ is an isometric isomorphism from the $\mathrm{JBW}^{*}$-triple $B$ into the $\mathrm{JBW}^{*}$-triple $A$. By [12, Proposition 2.2], $P^{*} e^{A}(x)$ is equal to $e^{B}(x)$ for all elements $x$ in $P A_{*}$. If $x$ is in $P A_{*}$ then, by [12, Lemma 2.1], $P^{*} e^{A}(x)-e^{A}(x)$ belongs to $D$. Therefore, $Q e^{B}(x)$ equals $e^{A}(x)$ for all elements $x$ in $P A_{*}$. Since the range of the restriction of $Q$ to $B$ contains $\left\{e^{A}(x): x \in P A_{*}\right\}$ and since $Q$ is weak* continuous, $Q$ clearly maps onto $C$. Therefore $C$ is a $\mathrm{JBW}^{*}$-subtriple of $A$. Moreover, being an M-sum of $\mathrm{JBW}^{*}$-subtriples $C \oplus_{M} D$ is a JBW*-triple and both (i) and (ii) follow immediately using [2] and [14, Lemma 4.4].

To prove (iii), observe that if $v$ is a tripotent in $A$ which is orthogonal to $e^{A}(x)$ for every element $x$ in $P A_{*}$ then, by the separate weak ${ }^{*}$ continuity of the triple product, $v$ is orthogonal to each tripotent in $C$. Since $D$ is a weak* closed subtriple in $A$ it follows that $D$ is contained in the intersection of the family $\left(A_{0}(u)\right)_{u \in \mathscr{U}(C)}$. The converse is obvious.

Attention is now turned to the special situation in which the projection $P$ occurring in Lemma 5.1 is neutral. 
LEMMA 5.2. Let $A$ be a $\mathrm{JBW}^{*}$-triple, let $P$ be a neutral projection on the predual $A_{*}$ of $A$, let $B$ be the range of the weak* continuous projection $P^{*}$ on $A$ and let the weak* closed subtriple $C$ and the weak* closed subtriple $D$ of $A$ be defined as above.

(i) Let $u$ be a tripotent in $A$. Then $u$ is an element in $C$ if and only if the set $\left\{x \in A_{*}:\|x\|=u(x)\right\}$ lies in the range $P A_{*}$ of $P$.

(ii) Let $p$ be a tripotent in the $\mathrm{JBW}^{*}$-triple $B$. Then the weak* limit $u(p)$ of the weak* convergent sequence $\left(p^{2 n+1}\right)$ is a tripotent in the $\mathrm{JBW}^{*}$-subtriple $C$ of $A$. Moreover, for all elements $x$ in $P A_{*}$, we have

$$
u\left(e^{B}(x)\right)=e^{A}(x)
$$

where $e^{B}(x)$ is the support of $x$ in the $\mathrm{JBW}^{*}$-triple $B$ and $e^{A}(x)$ is the support of $x$ in the JBW*-triple $A$.

(iii) The weak* closed subtriple $W(B)$ of $A$ generated by $B$ is contained in $C \bigoplus_{M} D$ and contains $C$ as a weak* closed ideal. Moreover, the restriction to $B$ of the $\mathrm{M}$-projection on $W(B)$ onto $C$ is an isometric isomorphism.

Proof. The same notation as that used in the proof of Lemma 5.1 is maintained. Let $u$ be a tripotent in $A$. If the set $\left\{x \in A_{*}:\|x\|=u(x)\right\}$ lies in $P A_{*}$ then, by [12, Lemma 2.5] and its proof, the tripotent $u$ is an element in $C$. Conversely, let $u$ be a tripotent in $C$. Let $x$ be an element in $A_{*}$ of norm one such that $u(x)$ is equal to one. By Lemma 5.1 (iii),

$$
P^{*} u=Q P^{*} u+(I-Q) P^{*} u=u+(I-Q) P^{*} \in u+A_{0}(u)_{1}
$$

Therefore $\left(P^{*} u\right)(x)$ is equal to one. Since $P^{*} u$ is an element in $B$ it follows that $x$ belongs to $B^{\sharp} \cap A_{*}$ and Lemma 3.1 shows that $x$ is an element in $P A_{*}$.

Let $p$ be a tripotent in the $\mathrm{JBW}^{*}$-triple $B$. By [8, Lemma 3.5], $u(p)$ is a tripotent in $A$ and $p$ is an element in the set $u(p)+A_{0}(u(p))_{1}$. If $x$ is an element of norm one in $A_{*}$ such that $u(p)(x)$ is equal to one then it follows that $p(x)$ is also equal to one and therefore, by an argument similar to the above, $x$ belongs to $P A_{*}$. By (i), $u(p)$ is an element in $C$. This proves the first part of (ii).

Let $x$ be an element in $P A_{*}$. Then

$$
e^{B}(x)=e^{A}(x)+(I-Q) e^{B}(x) \in e^{A}(x)+A_{0}\left(e^{A}(x)\right)_{1}
$$

Let $y$ be an element in $A_{*}$ of norm one. Clearly, if $e^{A}(x)(y)$ is equal to one, then so also is $e^{B}(x)(y)$. Conversely, if $e^{B}(x)(y)$ is equal to one then $y$ belongs to $B^{\sharp} \cap A_{*}$ and $y$ is an element in $P A_{*}$. Consequently, $y$ vanishes on $D$ and therefore $e^{A}(x)(y)$ is equal to one. It now follows, by [8, Lemma 3.4], that $u\left(e^{B}(x)\right)$ and $e^{A}(x)$ are equal, thereby proving (ii).

Let $W(B)$ be the smallest weak* closed subtriple of $A$ containing $B$. Since $B$ is contained in $C \oplus_{M} D$, it can be seen that $W(B)$ is contained in $C \oplus_{M} D$. Therefore, $C=Q(B) \subseteq Q(W(B)) \subseteq C$. By (ii), $C$ is a subset of $W(B)$ and it follows that $W(B)=C \oplus_{M}(I-Q)(W(B))$. Using [14, Lemma 4.4], $C$ is a weak* closed ideal in $W(B)$. The restriction to $W(B)$ of the map $Q$ is clearly an M-projection on $W(B)$ with range $C$. 
TheOREM 5.3. Let $A$ be a $\mathrm{JBW}^{*}$-triple with predual $A_{*}$ and let $B$ be an $\mathrm{N}^{*}$-ideal in $A$. $B, b y$

(i) When endowed with the triple product $\{\ldots\}_{B}$ defined, for elements $a, b$ and $c$ in

$$
\{a b c\}_{B}=P^{*}\{a b c\}
$$

where $P$ is the unique neutral projection on $A_{*}$ such that $B$ is the range of $P^{*}$, we have that $B$ is a $\mathrm{JBW}^{*}$-triple.

(ii) Let $C(B)$ be the weak* closed subspace of $A$ generated by the set

$$
\{u(p): p \in \mathscr{U}(B)\}
$$

where $u(p)$ is the weak* limit of the weak* convergent sequence $\left(p^{2 n+1}\right)$ in $A$. Then $C(B)$ is a complemented subtriple of $A$.

(iii) The $\mathrm{JBW}^{*}$-triple $C(B)$ is a weak* closed ideal in the weak* closed subtriple $W(B)$ of $B$ generated by $B$.

(iv) The restriction $R$ to $B$ of the M-projection from $W(B)$ onto $C(B)$ is an isometric isomorphism from the $\mathrm{JBW}^{*}$-triple $B$ onto the complemented subtriple $C(B)$ of $A$.

(v) The structural projection corresponding to $C(B)$ is $R P^{*}$.

Proof. By Lemma 5.1 and Lemma 5.2 it remains to prove (v) and that $B$ is a complemented subtriple of $A$. Since $C(B)$ is the smallest weak* closed subspace of $A$ containing the set $\left\{e^{A}(x): x \in P A_{*}\right\}$, it follows that $P A_{*}$ is a subset of $C(B)^{\sharp} \cap A_{*}$. Conversely, if $x$ is an element in $C(B)^{\#} \cap A_{*}$ then there exists a tripotent $u$ in the $\mathrm{JBW}^{*}$-subtriple $C(B)$ such that $u(x)$ equals $\|x\|$. By Lemma 5.2(i), $x$ lies in $P A_{*}$. Therefore the sets $P A_{*}$ and $C(B)^{\sharp} \cap A_{*}$ coincide and $C(B)^{\sharp} \cap A_{*}$ is a subspace.

Let $z$ be an element in the predual $C(B)_{*}$ of $C(B)$. The functional $x$ defined, for elements $a$ in $A$, by

$$
x(a)=\left(\left(R P^{*}\right) a\right)(z)
$$

is clearly a weak* continuous linear extension to $A$ of $z$. From the proof of Lemma 5.2 it can be seen that $R$ is the restriction to $B$ of the M-projection $Q$. It follows that $R P^{*}$ is a norm non-increasing projection with range $C(B)$. This shows that $C(B)$ is an $\mathrm{N}^{*}$-ideal and therefore, by Theorem 4.7 , a complemented subtriple.

Finally observe that, by Lemma 4.6 ,

$$
\begin{aligned}
\operatorname{Ker}(C(B)) & =\bigcap_{u \in \mathscr{Q}(C)}\left(A_{1}(u) \oplus A_{0}(u)\right)=\left(\bigcup_{u \in \mathscr{U}(C)} A_{2}(u)_{*}\right)^{\circ} \\
& =\left(\left(C^{\sharp} \cap A_{*}\right)^{\circ}=\left(B^{\sharp} \cap A_{*}\right)^{\circ}=\operatorname{ker} P^{*}=\operatorname{ker}\left(R P^{*}\right) .\right.
\end{aligned}
$$

The range of the projection $R P^{*}$ is $C(B)$ and (v) now follows from Theorem 4.5.

Acknowledgements. The authors are grateful for the support for their research has received from the United Kingdom Science and Engineering Research Council and the Schweizerische Nationalfonds/Fonds national suisse.

\section{References}

1. E. M. AlfSEn and E. G. EFrros, 'Structure in real Banach spaces I', Ann. of Math. 96 (1972) 98-128.

2. T. J. BARTON and R. M. TIMONEY, 'Weak* continuity of Jordan triple products and its applications', Math. Scand. 59 (1986) 177-191.

3. T. J. Barton, T. Dang and G. HoRN, 'Normal representations of Banach Jordan triple systems', Proc. Amer. Math. Soc. 102 (1987) 551-555. 
4. F. Cunningham JR, E. G. Effros and N. M. Roy, 'M-structure in dual Banach spaces', Israel J. Math. 14 (1973) 304-309.

5. S. Dineen, 'Complete holomorphic vector fields in the second dual of a Banach space', Math. Scand. 59 (1986) 131-142.

6. S. DiNEEN, 'The second dual of a JB*-triple system', Complex analysis, functional analysis and approximation theory (North-Holland, Amsterdam, 1986) 67-69.

7. C. M. Edwards, 'On Jordan W*-algebras', Bull. Sci. Math. (2) 104 (1980) 393-403.

8. C. M. EDWARDS and G. T. RütTIMANN, 'On the facial structure of the unit balls in a JBW*-triple and its predual', J. London Math. Soc. 38 (1988) 317-322.

9. C. M. Edwards and G. T. Rüttrimann, 'A characterization of inner ideals in JB*-triples', Proc. Amer. Math. Soc. 116 (1992) 1049-1057.

10. Y. Friedman and B. Russo, 'Structure of the predual of a JBW*-triple', J. Reine Angew. Math. 356 (1985) 67-89.

11. Y. Friedman and B. Russo, 'The Gelfand-Naimark theorem for JB*-triples', Duke Math. J. 53 (1986) 139-148.

12. Y. FRIEDMAN and B. Russo, 'Conditional expectation and bicontractive projections on Jordan C*-algebras and their generalizations', Math. Z. 194 (1987) 227-236.

13. Y. Friedman and B. Russo, 'Affine structure of facially symmetric spaces', Math. Proc. Cambridge Philos. Soc. 106 (1989) 107-124.

14. G. HoRn, 'Characterization of the predual and the ideal structure of a JBW*-triple', Math. Scand. 61 (1987) 117-133.

15. N. JACOBSON, Structure and representation of Jordan algebras, American Mathematical Society Colloquium Publications 39 (American Mathematical Society, Providence, 1968).

16. W. KAUP, 'A Riemann mapping theorem for bounded symmetric domains in complex Banach spaces', Math. Z. 183 (1983) 503-529.

17. O. Loos, 'On the socle of a Jordan pair', Collect. Math. 40 (1989) 109-125.

18. O. Loos and E. NeHER, 'Complementation of inner ideals in Jordan pairs', J. Algebra 166 (1994) 255-295.

19. K. MCCRimmon, 'Inner ideals in quadratic Jordan algebras', Trans. Amer. Math. Soc. 159 (1971) 445-468.

20. E. NEHER, Jordan triple systems by the grid approach, Lecture Notes in Mathematics 1280 (Springer, Berlin-Heidelberg-New York; 1987).

21. A. E. TAYLOR, 'The extension of a linear functional', Duke Math. J. 5 (1939) 538-547.

22. J. D. M. Wright, 'Jordan C*-algebras', Michigan Math. J. 24 (1977) 291-302.

23. M. A. Youngson, 'A Vidav theorem for Banach Jordan algebras', Math. Proc. Cambridge Philos. Soc. 84 (1978) 263-272.

The Queen's College

Oxford
University of Berne

Berne

Switzerland 